\title{
Dynamic Phasor Analysis and Design of Phase-Locked Loops for Single Phase Grid Connected Converters
}

\author{
Mohamed Rashed, Christian Klumpner and Greg Asher \\ Department of Electrical and Electronic Engineering, \\ The University of Nottingham, Nottingham, UK \\ e-mail: mohamed.rashed@nottingham.ac.uk
}

\begin{abstract}
Purpose - The purpose of the paper is to introduce the Dynamic Phasor Modelling (DPM) approach for stability investigation and control design of single-phase Phase Locked Loops PLLs. The aim is to identify the system instabilities not predicted using the existent analysis and design methods based on the simplified average model approach.

Design/methodology/approach - This paper starts by investigating the performance of three commonly used PLL schemes: the Inverse Park-PLL, the SOGI-Frequency-Locked-Loop and the Enhanced-PLL, designed using the simplified average model and will show that following this approach, there is a mismatch between their actual and desired transient performance. A new PLL design method is then proposed based on the DPM approach that allows the development of fourthorder DPM models. The small-signal eigenvalues analysis of the $4^{\text {th }}$ order DPM models is used to determine the control gains and the stability limits.

Findings - The DPM approach is proven to be useful for single-phase PLLs stability analysis and control parameters design. It has been successfully used to design the control parameters and to predict the PLL stability limits, which have been validated via simulation and experimental tests consisting of grid voltage sag, phase jump and frequency step change.

Originality/value - this paper has introduced the use of DPM approach for the purpose of singlephase PLL stability analysis and control design. The approach has enabled accurate control gains design and stability limits identification of single-phase PLLs.
\end{abstract}

Keywords Single phase converters, Phase locked Loop, PLL, Dynamic phasor analysis.

Paper type Research paper. 


\section{1- Introduction}

Phase-locked loops (PLLs) are widely used for interfacing power electronic converters to single and three-phase grids, (Chung, 2000; Golestan et al., 2013; Silva et al., 2004; Velasco et al., 2011). They are used to extract the information about the fundamental voltage component (phase angle, frequency and voltage magnitude) under various grid disturbances such as the steady state presence of unbalance and harmonics or transients: voltage sag, phase-jump and frequency change. The operation of converters in single phase systems is more challenging because of reduced level of information available in a single phase voltage compared to multiphase.

There are a few single-phase PLL schemes widely discussed in the literature that differ in their structure and estimation laws: the Inverse Park-PLL (IP-PLL) (Filho et al., 2008; Rashed et al., 2013), the Synchronous Reference Frame PLL (SRF-PLL) (Nicastri et al., 2010), the Second-Order Generalized Integrators (SOGI)-based Frequency-Locked Loop (FLL) (SOGI-FLL) (Rodr'iguez et al., 2011), the D-filter-based estimation PLL (Shinnaka, 2011), the Enhanced PLL (EPLL) (KarimiGhartemani, 2013; Karimi-Ghartemani et al., 2012) and the Modified Mixer Phase-Detector based PLL (MMPD-PLL), (Thacker et al., 2011). Some of this research work has been aimed at studying the design and performance analysis of single-phase PLLs. The design is typically performed using the simplified average model of the PLL (Karimi-Ghartemani, 2013; Karimi-Ghartemani et al., 2012; Thacker et al., 201; Freijedo et al., 2009), which ignores the effect of inherently generated doublefrequency component during transient on PLL stability. In (Karimi-Ghartemani, 2013), a comprehensive analysis and comparison of many single-phase PLL schemes is carried out using the simplified average model. The study concluded that the small signal mathematical model and the performance of the different PLL schemes were fairly similar, a conclusion which this paper will challenge.

This paper proposes a modelling technique not previously used in PLL stability analysis and design. The technique is known by Dynamic Phasor Modelling DPM and is suitable to represent and to predict the single-phase-PLL dynamic and instability modes not seen by the conventional average modelling technique used in the literature. In the DPM approach, the time-response of the system state variables is represented by a selective number of relevant frequency components of a Fourier series with slowly 
time-varying coefficients, (Stankovic et al., 1999; Sanders et al., 1991; Emadi, 2004; Caliskan et al., 1999; Mattavelli et al., 1999). The DPM approach has been successfully applied for modelling and analysis of single phase induction motors (Stankovic et al., 1999), PWM converters, (Sanders et al., 1991), diode bridge rectifiers (Emadi, 2004), DC/DC converters, (Caliskan et al., 1999) and thyristor controlled series capacitor compensators in power systems (Mattavelli et al., 1999).

The DPM approach is also used for the design and stability study of frequency and voltage droop control of microgrids, (Mariani et al., 2014; Xianwei et al., 2011; De Brabandere et al., 2005; Wang et al., 2012). The DPM is found effective in predicting system instabilities not seen by the conventional quasi-steady-state small signal model, (De Brabandere et al., 2005; Wang et al., 2012).

- In this paper, a $4^{\text {th }}$ order DPM is proposed and used for stability analysis, control design and performance comparison of the three representative single phase PLL schemes: the IP-PLL, SOGI-FLL and EPLL. The analysis will demonstrate the shortcomings of the conventional simplified average modelling for determining the stability limits and control gain design of the single-phase PLLs. The contribution of this paper lies in the following: Introducing the DPM approach for the purpose of single-phase PLLs stability analysis and control design.

- Accurate stability limits identification and control gains design of single-phase PLLs using DPM approach.

The paper is organised in five sections. Section 2 gives the basics of the DPM and PLLs. The design of the three PLL schemes using the simplified average model is presented in section 3. In section 4, the simulation results of the PLL schemes under investigation are used to show the discrepancy between their actual dynamic characteristics and the desired performance, and hence the inadequacy of the simplified average model based design. Section 5 details the proposed $4{ }^{\text {th }}$-order DPM small-signal stability analysis, design and comparison of the three PLLs. Large signal disturbance investigation and performance comparison of the three PLL schemes are presented in section 6 using simulation and experimental validation. Conclusions are given in section 7 .

\section{Fundamental Principles of DPM and PLL}


In this section, the fundamentals of the dynamic phasor modelling and the single-phase PLL concepts will be presented.

\subsection{Fundamentals of DPM}

In dynamic phasor modelling approach, the Fourier series coefficients of system state-variables are considered the DPM system-state-variables and the state equations are derived for these Fourier coefficients. Therefore, a system state variable $x(\tau)$ can be represented on the interval $\tau \in((\mathrm{t}-\mathrm{T}), \mathrm{t}]$ using a Fourier series of the form, (Stankovic et al., 1999):

$$
x(\tau)=\sum_{k=-\infty}^{\infty}\left[\langle x\rangle_{k}(t)\right] e^{j k \omega_{b} \tau}
$$

Where, $\mathrm{T}$ is the time period for the base frequency, $\omega_{\mathrm{b}}=2 \pi / \mathrm{T},\langle x\rangle_{k}(t)$ is the $\mathrm{k}^{\text {th }}$ complex Fourier coefficient that is varying with time since the interval under consideration slides with time t. The notation $<>$ denotes the averaging operation that is applied to determine the $\mathrm{k}^{\text {th }}$ complex Fourier coefficient at time $t$. The averaging operation is

$$
\langle x\rangle_{k}(t)=\frac{1}{T} \int_{t-T}^{t} x(\tau) e^{j k \omega_{b} \tau} d \tau
$$

The $\mathrm{k}^{\text {th }}$-order DPM state-space differential equation is a state-space equation formed for the $\mathrm{k}^{\text {th }}$ frequency-order Fourier series coefficient of the system state variables. The Zero-Order (ZO) DPM equation is the system state-space equation for the dc coefficients of the Fourier series for the system state variables. In practice, the ZO-DPM is the simplified average model usually used in the literature for conventional PLL design.

The derivative of the $\mathrm{k}^{\text {th }}$ complex Fourier coefficient (2) is given by:

$$
\frac{d}{d t}\left(\langle x\rangle_{k}\right)=\left\langle\frac{d}{d t} x\right\rangle_{k}-j k \omega_{b}\langle x\rangle_{k}
$$

Also, the $\mathrm{k}^{\text {th }}$ Fourier coefficient for a nonlinear term (e.g. a product of two state variables $x$ and $y$ ) can be obtained using the convolution property (Sanders et al., 1991) as follows:

$$
\langle x y\rangle_{k}=\sum_{l=-\infty}^{\infty}\langle x\rangle_{(k-l)}\langle y\rangle_{l}
$$

Noting that the phasor $\langle x\rangle_{-k}$ is the complex conjugate of $\langle x\rangle_{k}$. The properties in (3) and (4) are essential for deriving the PLL DPM from the time-domain state space model.

This mathematical approach will be used later to derive the DPM for the PLL schemes under study.

\subsection{Fundamentals of PLLs}


The structure of a typical PLL scheme that includes also the single-phase PLLs is originated from the well-established three-phase SRF-PLL (Chung, 2000). The 3-phase SRF-PLL contains three main components: the Phase Detector (PD), the filter (which is usually a PI controller), and the Voltage Controlled Oscillator (VCO). These components aim at synchronising the PLL estimated output voltage vector with the input voltage vector as represented by the two orthogonal $\alpha \beta$ voltage components as illustrated in the phasor diagram shown in Fig. 1. The PD generates an error signal (referred as "adaptive law") that is proportional to the phase difference between the input and the estimated output voltage vectors. The error signal is utilised to modify the frequency of the VCO until the average frequency and the phase angle of the input and the estimated (output) voltage vectors are equal (Golestan et al., 2013).

In single phase PLLs (e.g. Fig.2), the measured grid voltage is fed as the $\alpha$-axis component of the input voltage vector while the $\beta$-axis is substituted by a virtual voltage component. The virtual $\beta$-axis voltage component can be constructed by applying a $90^{\circ}$ phase shift to the measured $\alpha$-axis voltage component (Silva et al., 2004; Velasco et al., 2011) or substituted by the estimated $\beta$-axis component, (Filho et al., 2008; Rodr'iguez et al., 2011; Karimi-Ghartemani, 2013). The main problem affecting the performance of a single phase PLL is that any mismatch/error in the virtual $\beta$-axis component (during transient or steady state) will produce double-frequency ripple component that adversely affects the dynamic performance and the stability of the PLL.

The relationships between the input and the estimated output voltage vectors and their phase angles and rotational speeds are represented and defined by the phasor diagram shown in Fig. 1. 


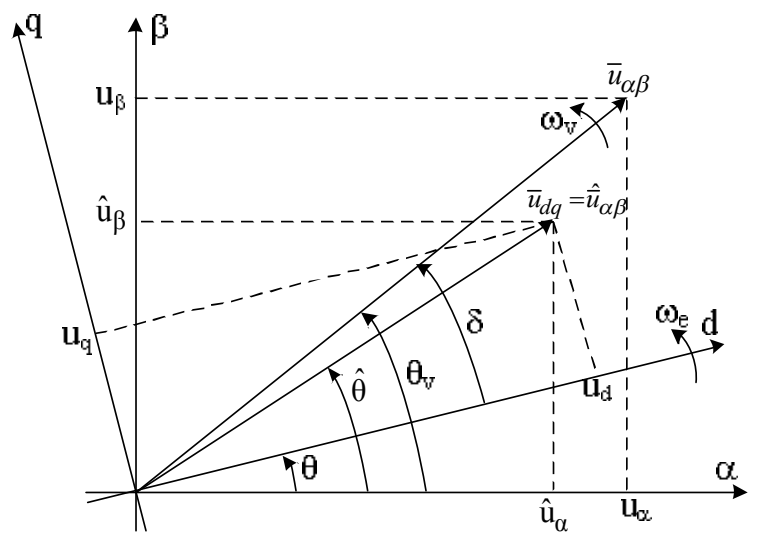

Fig. 1 Single-phase PLL phasor diagram.

Where $\mathrm{u}_{\alpha}$ is the measured grid voltage whilst $\bar{u}_{\alpha \beta}=u_{\alpha}+j u_{\beta}$ is the fictious $\alpha \beta$ grid voltage vector. If $U_{v}$ is the grid voltage magnitude, then $u_{\alpha}=U_{v} \cos \left(\theta_{v}\right)$. The estimated (output) voltage vector $\hat{u}_{\alpha \beta}=\hat{u}_{\alpha}+j \hat{u}_{\beta}=\bar{u}_{d q}$ has the corresponding $\mathrm{u}_{\mathrm{d}}, \mathrm{u}_{\mathrm{q}}$ components in the dq rotating reference frame. The corresponding angles and the rotational speeds that will be used in the development of the DPM of the PLL are also shown in Fig. 1. The phase angle $\delta$ is the phase angle difference between the phase angle of the input voltage vector, $\theta_{v}$ and the phase angle $\hat{\theta}$.

\section{Modelling and Control Design of Single-Phase PLLs}

In this section, the IP-PLL, (Filho et al., 2008), SOGI-FLL, (Rodr'iguez et al., 2011) and the EPLL (Karimi-Ghartemani, 2013; Karimi-Ghartemani et al., 2012) single-phase PLL schemes will be modelled and the phase angle, voltage magnitude and frequency estimation algorithms for the three PLLs will be established utilising the simplified average model. This will be used in the next sections to prove that the dynamic performance of the designed PLLs will not match with the design specification.

The convention used in this paper is that the input of the PLL seen as a control system is the grid voltage, while the outputs are the estimated grid voltage magnitude, frequency and phase angle, which are defined as $\mathrm{u}_{\text {out }}, \omega_{\text {out }}$ and $\theta_{\text {out }}$, independent on the estimation method used in each PLL. 


\subsection{The Modelling and Control Design of the Inverse Park PLL}

The typical model of the Inverse Park PLL (IP-PLL) expressed in rotating reference frame (Filho et al., 2008; Karimi-Ghartemani, 2013) is shown in Fig. 2.

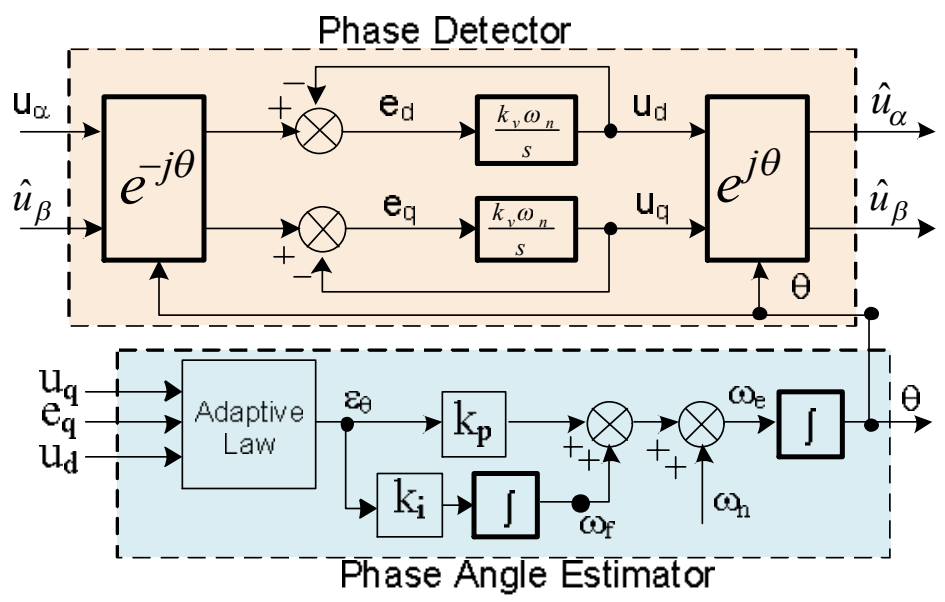

Fig. 2 The model of the Inverse Park Phase Locked Loop (IP-PLL)

The state space variable model for the IP-PLL (Fig. 2) in the rotating reference frame $d q$ is given by:

$$
\begin{aligned}
& \dot{u}_{d}=k_{v} \omega_{n}\left[0.5 U_{v} \cos (\delta)-0.5 u_{d}+0.5 U_{v} \cos (\delta+2 \theta)-0.5 u_{d} \cos (2 \theta)+0.5 u_{q} \sin (2 \theta)\right] \\
& \dot{u}_{q}=k_{v} \omega_{n}\left[0.5 U_{v} \sin (\delta)-0.5 u_{q}-0.5 U_{v} \sin (\delta+2 \theta)+0.5 u_{d} \sin (2 \theta)+0.5 u_{q} \cos (2 \theta)\right] \\
& \dot{\omega}_{f}=k_{i}\left(\varepsilon_{\theta}\right) \\
& \dot{\delta}=\omega_{v}-k_{p} \varepsilon_{\theta}-\omega_{f}-\omega_{n}
\end{aligned}
$$

Where: $\varepsilon_{\theta}$ is the adaptive law (error signal) given by (6), and $\omega_{e}=\left(k_{p} \varepsilon_{\theta}+\omega_{f}+\omega_{n}\right), \delta=\theta_{v}-\theta$, $\frac{d}{d t} \theta=\omega_{e}, \frac{d}{d t} \theta_{v}=\omega_{v}$ and $\omega_{\mathrm{n}}$ is angular speed ( $\left.\mathrm{rad} / \mathrm{s}\right)$ corresponding to the nominal grid frequency $(50 \mathrm{~Hz})$.

The adaptive law $\varepsilon_{\theta}$ for IP-PLL phase angle estimation was obtained as in (Filho et al., 2008; Rashed et al., 2013):

$$
\varepsilon_{\theta}=\frac{u_{q}}{u_{d}}
$$

It should be noted that in the literature, the simplified average model typically used in PLL design (Filho et al., 2008; Karimi-Ghartemani, 2013; Thacker et al., 2011; Freijedo et al., 2009) is obtained by ignoring the double-frequency sine and cosine terms in $(5 a-b)$.

For the development of the dynamic-phasor differential equations used in the modelling of the PLL schemes under study in this paper for the purpose of stability analysis and control design, the time 
period $\mathrm{T}$ in (1),(2) is set equal to $2 \pi /\left\langle\omega_{e}\right\rangle_{0}$ and hence $\omega_{b}$ in (3) is substituted by $\left\langle\omega_{e}\right\rangle_{0}$. Then, the generalised $\mathrm{k}^{\text {th }}$-order dynamic-phasor state-space differential equations for (5) are given by:

$$
\begin{aligned}
& \frac{d}{d t}\left\langle u_{d}\right\rangle_{k}=-j k\left\langle\omega_{e}\right\rangle_{0}\left\langle u_{d}\right\rangle_{k}+k_{v} \omega_{n}\left\langle e_{d}\right\rangle_{k} \\
& \frac{d}{d t}\left\langle u_{q}\right\rangle_{k}=-j k\left\langle\omega_{e}\right\rangle_{0}\left\langle u_{q}\right\rangle_{k}+k_{v} \omega_{n}\left\langle e_{q}\right\rangle_{k} \\
& \frac{d}{d t}\left\langle\omega_{f}\right\rangle_{k}=-j k\left\langle\omega_{e}\right\rangle_{0}\left\langle\omega_{f}\right\rangle_{k}+k_{i}\left\langle\varepsilon_{\theta}\right\rangle_{k} \\
& \frac{d}{d t}\langle\delta\rangle_{k}=-j k\left\langle\omega_{e}\right\rangle_{0}\langle\delta\rangle_{k}-\left\langle k_{p} \varepsilon_{\theta}+\omega_{f}+\omega_{n}\right\rangle_{k}+\left\langle\omega_{v}\right\rangle_{k}
\end{aligned}
$$

where, $e_{d}, e_{q}$ are

$$
\begin{aligned}
& e_{d}=0.5 U_{v} \cos (\delta)-0.5 u_{d}+0.5 U_{v} \cos (\delta+2 \theta)-0.5 u_{d} \cos (2 \theta)+0.5 u_{q} \sin (2 \theta) \\
& e_{q}=0.5 U_{v} \sin (\delta)-0.5 u_{q}-0.5 U_{v} \sin (\delta+2 \theta)+0.5 u_{d} \sin (2 \theta)+0.5 u_{q} \cos (2 \theta)
\end{aligned}
$$

The DPM of the nonlinear terms such as $\cos (2 \theta), \sin (2 \theta)$ and $\left(\mathrm{u}_{\mathrm{q}} / \mathrm{u}_{\mathrm{d}}\right)$ in $(7 \mathrm{e}, \mathrm{f}),(6)$ are obtained as in Appendix 1. The ZO-DPM that corresponds to $\mathrm{k}=0$ in (7) is then given by:

$$
\begin{aligned}
& \left\langle\dot{u}_{d}\right\rangle_{0}=k_{v} \omega_{n}\left(0.5\left\langle U_{v}\right\rangle_{0}-0.5\left\langle u_{d}\right\rangle_{0}\right) \\
& \left\langle\dot{u}_{q}\right\rangle_{0}=k_{v} \omega_{n}\left\langle 0.5 U_{v} \delta-0.5 u_{q}\right\rangle_{0} \\
& \left\langle\dot{\omega}_{f}\right\rangle_{0}=k_{i}\left\langle\varepsilon_{\theta}\right\rangle_{0} \\
& \langle\dot{\delta}\rangle_{0}=\left\langle\omega_{v}\right\rangle_{0}-\left\langle k_{p} \varepsilon_{\theta}+\omega_{f}+\omega_{n}\right\rangle_{0}
\end{aligned}
$$

and

$$
\begin{aligned}
& \left\langle e_{d}\right\rangle_{0}=0.5\left\langle U_{v}\right\rangle_{0}-0.5\left\langle u_{d}\right\rangle_{0} \\
& \left\langle e_{q}\right\rangle_{0}=0.5\left\langle U_{v}\right\rangle_{0}\langle\delta\rangle_{0}-0.5\left\langle u_{q}\right\rangle_{0}
\end{aligned}
$$

From now on, unless otherwise mentioned, the averaging operation symbol $<>_{0}$ will be ignored for simplicity.

In the following sections, the ZO-DPM such as in (8), which is the simplified average model typically used in the literature in the PLL design, will be used for the design of the phase angle, voltage magnitude and frequency estimator to achieve the design specifications set for the small signal closed loop transfer function (CLTF) of $\left(\theta_{\text {out }} / \theta_{\mathrm{v}}\right),\left(\mathrm{u}_{\text {out }} / \mathrm{U}_{\mathrm{v}}\right)$ and $\left(\omega_{\text {out }} / \omega_{\mathrm{v}}\right)$ for all PLL schemes under study. Afterwards, the actual dynamic performance of the designed PLLs will be proven not to match the design specifications and hence proving the shortcoming of using the ZO-DPM for single-phase PLL design.

First, the small signal ZO-DPM for the adaptive law (6) is derived in Laplace form using (8) for the PLL equilibrium point where $\left[u_{d} u_{q}\right]=\left[U_{v} 0\right]$ : 


$$
\Delta \varepsilon_{\theta}=\frac{0.5 k_{v} \omega_{n}}{s+0.5 k_{v} \omega_{n}} \Delta \delta
$$

(9) shows that $\Delta \varepsilon_{\theta}$ is linearly dependent on $\Delta \delta$ via the transfer function of a LPF. To eliminate the LPF influence in (9), a new phase angle adaptive law to replace (6) is introduced in this paper:

$$
\varepsilon_{\theta}=\frac{u_{q}}{u_{d}}+2\left(\frac{e_{q}}{u_{d}}\right)
$$

which results in a small signal ZO-DPM of

$$
\Delta \varepsilon_{\theta}=\Delta \delta
$$

identical (for comparison purpose) to the small signal adaptive law model of the other PLL scheme (EPLL) as it will be shown later. Fig. 3 depicts the resulting small signal ZO-DPM for the IP-PLL phase angle estimator using the proposed adaptive law in (10),(11).

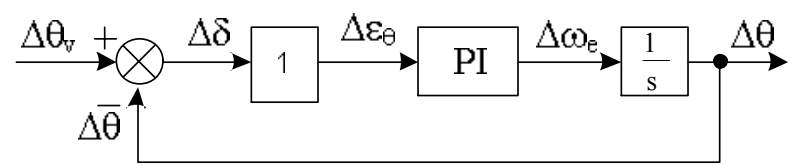

Fig. 3 Small signal ZO-DPM for the IP-PLL phase angle estimator

In the IP-PLL, the outputs $\theta_{\text {out }}$ and $\mathrm{u}_{\text {out }}$ are set equal to $\theta$ and $\mathrm{u}_{\mathrm{d}}$. Hence, the small signal ZO-DPM

CLTF for the IP-PLL phase angle estimator (from Fig. 3) is given by:

$$
\frac{\Delta \theta_{o u t}}{\Delta \theta_{v}}=\frac{\Delta \theta}{\Delta \theta_{v}}=\frac{k_{p} s+k_{i}}{s^{2}+k_{p} s+k_{i}}
$$

Where $\mathrm{k}_{\mathrm{p}}$ and $\mathrm{k}_{\mathrm{i}}$ are the gains of the PI controller.

The small signal CLTF for the output (estimated) voltage magnitude is also derived from (8) and is equivalent to a first order LPF (13) with a time constant of $2 / k_{v} \omega_{n}$ :

$$
\frac{\Delta u_{o u t}}{\Delta U_{v}}=\frac{\Delta u_{d}}{\Delta U_{v}}=\frac{0.5 k_{v} \omega_{n}}{s+0.5 k_{v} \omega_{n}}
$$

The gain $\mathrm{k}_{\mathrm{v}}(13)$ determines the dynamic response of the voltage magnitude estimation. On the other hand, the $\mathrm{k}_{\mathrm{p}}$ and $\mathrm{k}_{\mathrm{i}}$ gains of the PI controller (12) determine the dynamic characteristics for the phaseangle estimation. The values of $\mathrm{k}_{\mathrm{p}}$ and $\mathrm{k}_{\mathrm{i}}$ are chosen to achieve a damping coefficient $\xi=1$ for (12) as recommended in (Karimi-Ghartemani et al., 2012; Freijedo et al., 2009). In this paper, $\mathrm{k}_{\mathrm{p}}$ is selected to be equal to $k_{v} \omega_{n}$ so that the CLTF poles of (12) coincide with the CLTF pole of the voltage magnitude estimator in (13). And hence, $k_{i}=\left(k_{v} \omega_{n} / 2\right)^{2}$. Therefore, the small signal ZO-DPM CLTF poles of 
the PLL voltage and phase-angle estimators are located on the real axis at $\left(-k_{v} \omega_{n} / 2\right)$. Then, the phase-angle estimator small signal CLTF (13) can be expressed as:

$$
\frac{\Delta \theta_{\text {out }}}{\Delta \theta_{v}}=\frac{\Delta \theta}{\Delta \theta_{v}}=\frac{k_{v} \omega_{n} s+\left(0.5 k_{v} \omega_{n}\right)^{2}}{s^{2}+k_{v} \omega_{n} s+\left(0.5 k_{v} \omega_{n}\right)^{2}}
$$

In (Rodr'iguez et al., 2011), the small signal CLTF for the frequency estimator $\omega_{\text {out }} / \omega_{\mathrm{v}}$ was equivalent to a first order LPF. In this paper (for comparison purpose) we will also carry out the design to achieve a LPF behaviour for the CLTF of the frequency estimator. Therefore, $\omega_{\text {out }}$ for IP-PLL is proposed here to be:

$$
\omega_{\text {out }}=\omega_{e}-0.5 k_{p} \varepsilon_{\theta}
$$

which yields a LPF small signal ZO-DPM CLTF of;

$$
\frac{\Delta \omega_{0 u t}}{\Delta \omega_{v}}=\frac{0.5 k_{v} \omega_{n}}{s+0.5 k_{v} \omega_{n}}
$$

It should be noted from (13), (14) and (16) that the CLTF poles for the PLL estimators $\left(\theta_{\text {out }}, \mathrm{u}_{\text {out }}\right.$ and $\left.\omega_{\text {out }}\right)$ are located at $-0.5 \mathrm{k}_{\mathrm{v}} \omega_{\mathrm{n}}$ and $\mathrm{k}_{\mathrm{v}}$ becomes the only gain that determines the small signal dynamic response of the PLL estimators. Having only one control gain is deliberate to simplify the comparison of the PLLs in this paper.

In the next sections, small signal ZO-DPM CLTFs will be derived for the SOGI-FLL and EPLL estimators to be identical to (13), (14) and (16), which if the ZO-DPM design approach is adequate, it will result in identical performance matching the design specification.

\subsection{The Modelling and Control Design of the SOGI-FLL}

The typical implementation of the SOGI-FLL (Rodr'iguez et al., 2011) is shown in Fig. 4. Compared to the IP-PLL, SOGI-FLL model is implemented in the stationary reference frame. For comparison purpose, the stationary frame SOGI-FLL model needs to be transformed to the rotating reference frame. 


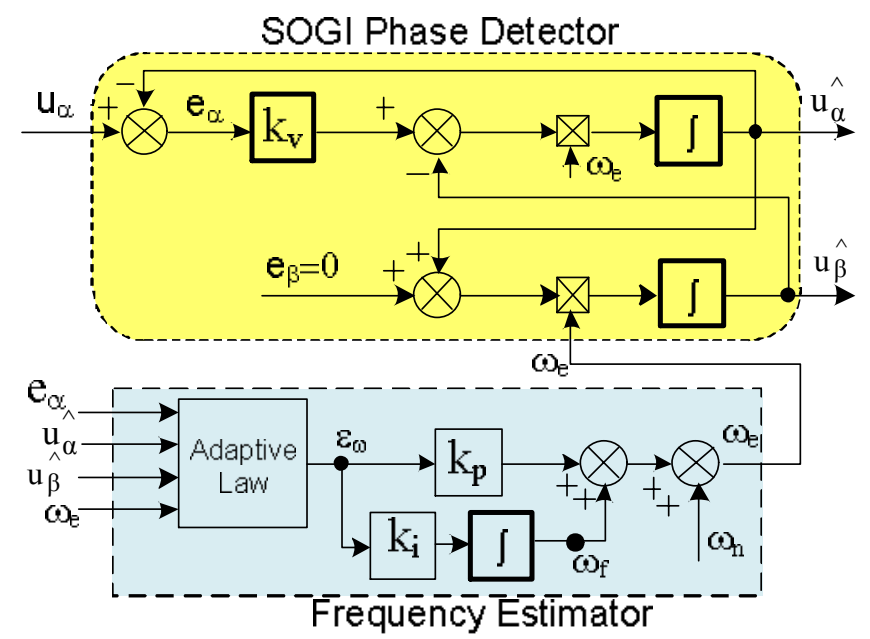

Fig. 4 Typical model of the SOGI-FLL

The model for the SOGI-FLL phase detector (Fig.4) as presented in (Rodr'iguez et al., 2011) is

$$
\dot{\hat{u}}_{\alpha}=\left[\left(u_{\propto}-\hat{u}_{\propto}\right) k_{v}-\hat{u}_{\beta}\right] \omega_{e} \quad \& \quad \dot{\hat{u}}_{\beta}=\omega_{e} \hat{u}_{\propto}
$$

The transformed SOGI-FLL (Fig. 4) model expressed in the rotating reference frame (assuming slow varying $\left.\omega_{\mathrm{e}}\right)$ is:

$$
\begin{aligned}
& \dot{u}_{d}=k_{v} \omega_{e}\left[0.5 U_{v} \cos (\delta)-0.5 u_{d}+0.5 U_{v} \cos (\delta+2 \theta)-0.5 u_{d} \cos (2 \theta)+0.5 u_{q} \sin (2 \theta)\right] \\
& \dot{u}_{q}=k_{v} \omega_{e}\left[0.5 U_{v} \sin (\delta)-0.5 u_{q}-0.5 U_{v} \sin (\delta+2 \theta)+0.5 u_{d} \sin (2 \theta)+0.5 u_{q} \sin (2 \theta)\right] \\
& \dot{\omega}_{f}=k_{i}\left(\varepsilon_{\omega}\right) \\
& \dot{\delta}=\omega_{v}-k_{p} \varepsilon_{\omega}-\omega_{f}-\omega_{n}
\end{aligned}
$$

where: $\varepsilon_{\omega}$ is the adaptive law and is given by (19), $\omega_{e}=\left(k_{p} \varepsilon_{\omega}+\omega_{f}+\omega_{n}\right)$ and $d \theta / d t=\omega_{e}$.

The model in (18) is quite similar to that of IP-PLL (5). However, the SOGI-FLL is robust to grid frequency variation since $\omega_{\mathrm{n}}$ in (5) is replaced by the estimated value $\omega_{\mathrm{e}}$ in (18).

The frequency adaptive law as given in (Rodr'iguez et al., 2011) is:

$$
\varepsilon_{\omega}=\frac{-k_{v} \omega_{e} e_{\alpha} \widehat{u}_{\beta}}{\widehat{u}_{\alpha}^{2}+\widehat{u}_{\beta}^{2}}
$$

The small-signal ZO-DPM based transfer function of the adaptive law in (19) around the equilibrium point $\left(\omega_{e}=\omega_{n}, u_{q}=0\right)$ is:

$$
\Delta \varepsilon_{\omega}=\frac{0.5 k_{v} \omega_{n}}{s+0.5 k_{v} \omega_{n}}(\Delta \dot{\delta})
$$

Equation (20) shows that contrary to the small signal model given in (Rodr'iguez et al., 2011), the adaptive law small signal transfer function is equivalent to the transfer function of a first order LPF and for this reason, a full PI controller is used (see Fig. 4) for frequency estimation rather than an 
Integral controller as in (Rodr'iguez et al., 2011). The PI controller gains are set $k_{p}=1$ and $k_{i}=$ $0.5 k_{\nu} \omega_{e}$ in order to obtain a small-signal CLTF equivalent to that of the IP-PLL (16). Hence, the small signal ZO-DPM for the frequency estimator (19)-(20) is represented by the block diagram in Fig. 5.

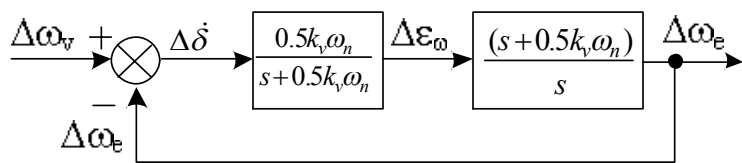

Fig. 5 Small signal ZO-DPM for the SOGI-FLL frequency estimator

with the small-signal ZO-DPM based CLTF:

$$
\frac{\Delta \omega_{\text {out }}}{\Delta \omega_{v}}=\frac{\Delta \omega_{e}}{\Delta \omega_{v}}=\frac{0.5 k_{v} \omega_{n}}{s+0.5 k_{v} \omega_{n}}
$$

The SOGI-FLL output phase angle $\theta_{\text {out }}$ is given by, (Rodr'iguez et al., 2011):

$$
\theta_{\text {out }}=\hat{\theta}=\operatorname{atan}\left(\frac{\widehat{u}_{\alpha}}{\widehat{u}_{\beta}}\right)=\theta+\operatorname{atan}\left(\frac{u_{q}}{u_{d}}\right)
$$

Then, the small signal ZO-DPM based CLTF for the phase angle estimator is derived using (21), (22)

and (18) at the equilibrium point $\left(\omega_{e}=\omega_{n}, u_{q}=0\right)$ and given by:

$$
\frac{\Delta \theta_{\text {out }}}{\Delta \theta_{v}}=\frac{\Delta \theta}{\Delta \theta_{v}}=\frac{k_{v} \omega_{n} s+\left(0.5 k_{v} \omega_{n}\right)^{2}}{s^{2}+k_{v} \omega_{n} s+\left(0.5 k_{v} \omega_{n}\right)^{2}}
$$

which is equivalent to (14) for the IP-PLL. Furthermore, the estimated voltage magnitude in SOGI-

FLL is calculated as

$$
u_{\text {out }}=\hat{u}=\sqrt{u_{d}^{2}+u_{q}^{2}}
$$

and the small signal ZO-DPM based CLTF for (24) at the equilibrium point $\left(\omega_{e}=\omega_{n} ; u_{q}=0\right)$ is derived and given by:

$$
\frac{\Delta u_{\text {out }}}{\Delta U_{v}}=\frac{\Delta \widehat{u}}{\Delta U_{v}}=\frac{0.5 k_{v} \omega_{n}}{s+0.5 k_{v} \omega_{n}}
$$

From (21),(23),(25), the SOGI-FLL is designed to provide identical small signal ZO-DPM CLTF to that for IP-PLL frequency (16), phase angle (14) and voltage magnitude (13) estimators. This procedure will be repeated for the EPLL in the next section.

\subsection{The Modelling and Control Design of the EPLL}

The typical EPLL model (Karimi-Ghartemani, 2013; Karimi-Ghartemani et al., 2012) is represented by the block diagram given in Fig. 6 . 


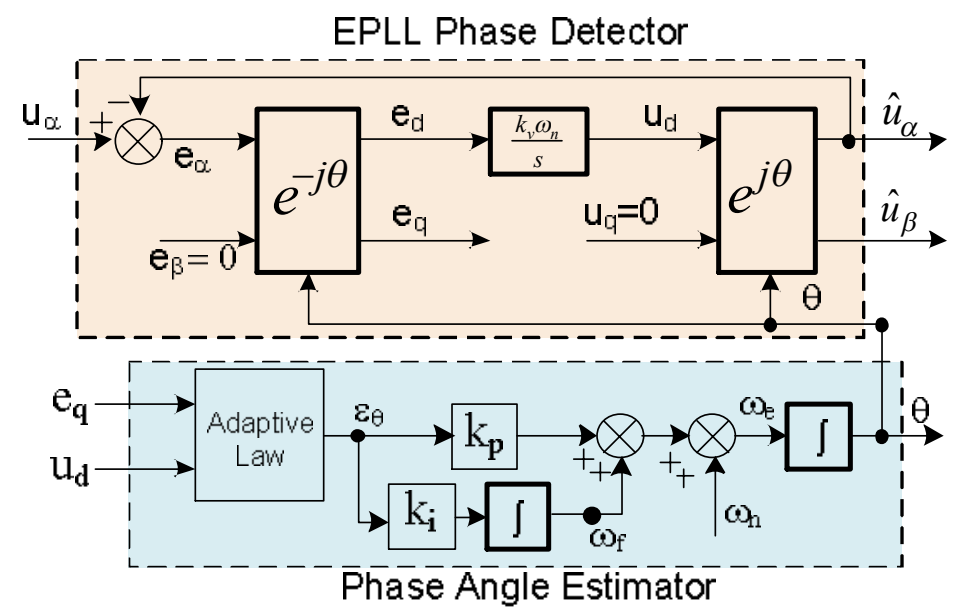

Fig. 6 Typical model of the EPLL

The EPLL model in the rotating reference frame is:

$$
\begin{aligned}
& \dot{u}_{d}=k_{v} \omega_{n}\left[0.5 U_{v} \cos (\delta)-0.5 u_{d}+0.5 U_{v} \cos (\delta+2 \theta)-0.5 u_{d} \cos (2 \theta)\right] \\
& \dot{\omega}_{f}=k_{i}\left(\varepsilon_{\theta}\right) \\
& \dot{\delta}=\omega_{v}-k_{p} \varepsilon_{\theta}-\omega_{f}-\omega_{n} \\
& e_{q}=\left[0.5 U_{v} \sin (\delta)-0.5 U_{v} \sin (\delta+2 \theta)+0.5 u_{d} \sin (2 \theta)\right]
\end{aligned}
$$

Where, the adaptive law $\varepsilon_{\theta}$ for phase angle estimation as used in (Karimi-Ghartemani, 2013; KarimiGhartemani et al., 2012) is:

$$
\varepsilon_{\theta}=\frac{2 e_{q}}{u_{d}}
$$

The small signal ZO-DPM transfer function for the adaptive law (27) at the equilibrium point $\left(\mathrm{e}_{\mathrm{q}}=0\right)$ is:

$$
\Delta \varepsilon_{\theta}=\Delta \delta
$$

which is identical to (11) for IP-PLL and hence $\mathrm{k}_{\mathrm{p}}, \mathrm{k}_{\mathrm{i}}$ and the ZO-DPM CLTF of the phase angle estimator for the EPLL are equal to that given in (12) and (14).

In the EPLL, $u_{\text {out }}=u_{d}$ and hence the small-signal ZO-DPM CLTF of the voltage magnitude estimator is derived from (26) and given by:

$$
\frac{\Delta u_{o u t}}{\Delta U_{v}}=\frac{\Delta u_{d}}{\Delta U_{v}}=\frac{0.5 k_{v} \omega_{n}}{s+0.5 k_{v} \omega_{n}}
$$

Similar to the IP-PLL, the output frequency $\omega_{\text {out }}$ for the EPLL is calculated as.

$$
\omega_{\text {out }}=\omega_{e}-0.5 k_{v} \omega_{n} \varepsilon_{\theta}
$$

with the small-signal ZO-DPM CLTF of:

$$
\frac{\Delta \omega_{\text {out }}}{\Delta \omega_{v}}=\frac{0.5 k_{v} \omega_{n}}{s+0.5 k_{v} \omega_{n}}
$$


with this, the small signal ZO-DPM CLTFs for phase angle, frequency and voltage magnitude estimators for all three PLL schemes under study have been designed to be identical and this should lead to identical dynamic performance. Also, the transfer functions show that the PLLs should remain stable in a very wide range of $\mathrm{k}_{\mathrm{v}}$. In the next section, the performance of the designed PLLs (using the ZO-DPM) will be investigated using simulations with different values of $\mathrm{k}_{\mathrm{v}}$ which should help in validating the ZO-DPM based design approach and identifying the potential differences in actual dynamic performance.

\section{Performance Comparison of PLL Schemes Designed Using the ZO-DPM}

The PLL schemes presented in Figs 2, 4 and 6 and using the adaptive laws in (10), (19) and (27) and having the control gains $\mathrm{k}_{\mathrm{p}}$ and $\mathrm{k}_{\mathrm{i}}$ designed to provide identical ZO-DPM based small signal CLTFs are implemented using Simulink/Matlab. The simulation models are tested for three small step changes in phase-angle, frequency and voltage magnitude for different values of gain $\mathrm{k}_{\mathrm{v}}$. The higher the value of $k_{v}$, the faster the expected PLL dynamic response.

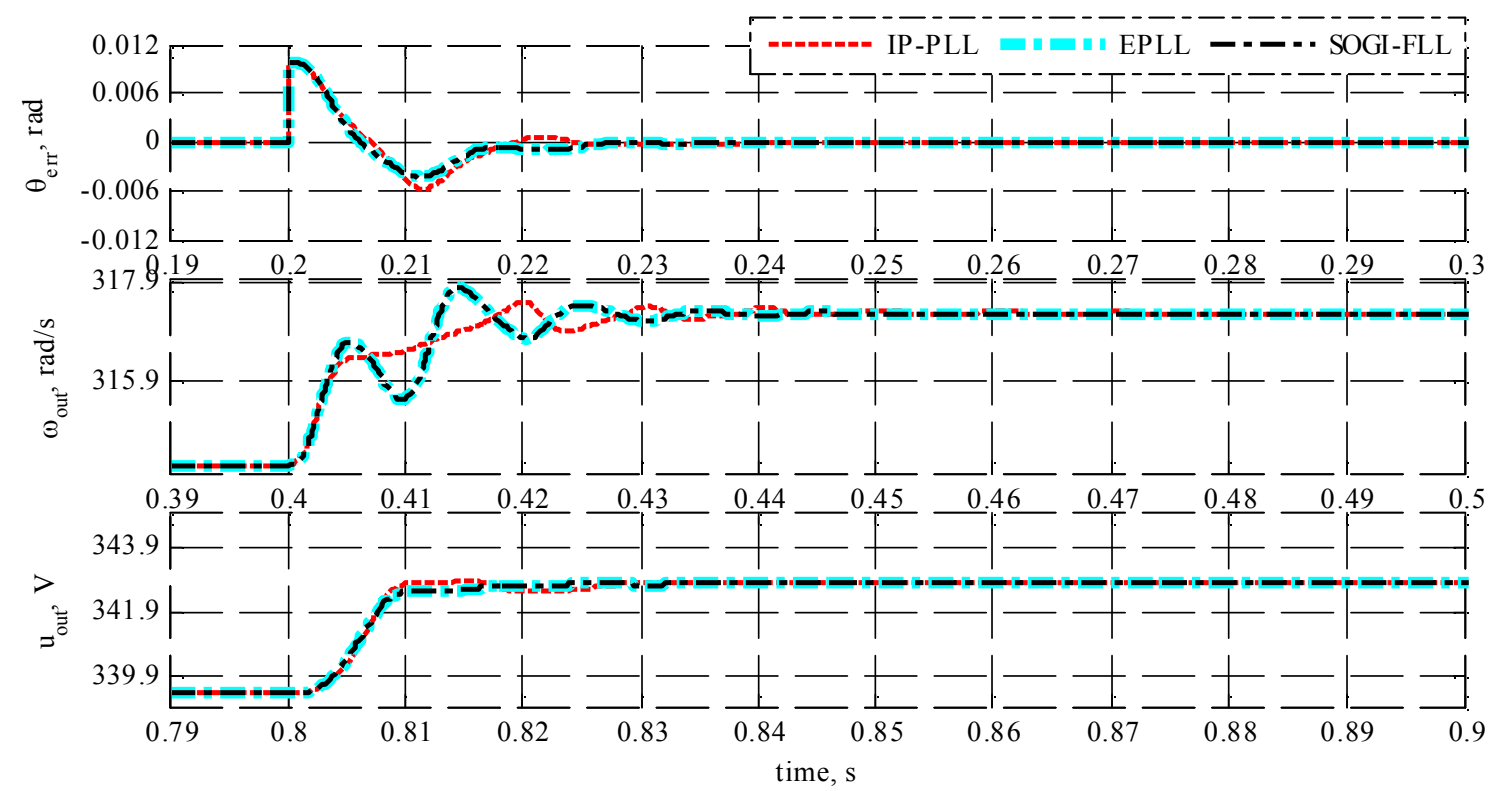

Fig. 7 Simulation results: PLLs testing under three small signal step changes for $\mathrm{k}_{\mathrm{v}}=1$. Top subplot: shows phase angle response to a phase-angle step of $0.01 \mathrm{rad}(\mathrm{at} \mathrm{t}=0.2 \mathrm{~s})$, middle subplot: shows frequency response to a frequency step of $1 \%$ (at $\mathrm{t}=0.4 \mathrm{~s}$ ), bottom subplot: shows voltage magnitude response to a voltage step of $1 \%$ (at $\mathrm{t}=0.8 \mathrm{~s})$. "red" IP-PLL, “cyan" EPLL, "black" SOGI-FLL.

The simulation results from the three tests with $\mathrm{k}_{\mathrm{v}}=1$ is shown in Fig. 7. It is noted that the dynamic response for all PLLs has a superimposed transient oscillation that decays quickly. The SOGI-FLL and the EPLL response are visibly identical for all three tests, while the IP-PLL response differs slightly, 
with the largest mismatch noticed in the frequency step change test. The three tests are repeated for $\mathrm{kv}$ $=2$ (faster PLL dynamic performance is expected). The simulation results are shown in Fig. 8. It is clear that the IP-PLL becomes unstable in all three tests but the SOGI-FLL and the EPLL remain stable but having slow decaying oscillations, although a higher $\mathrm{k}_{\mathrm{v}}$ should have resulted in shorter time response, which contradicts the desired dynamic performance set for the ZO-DPM based design.

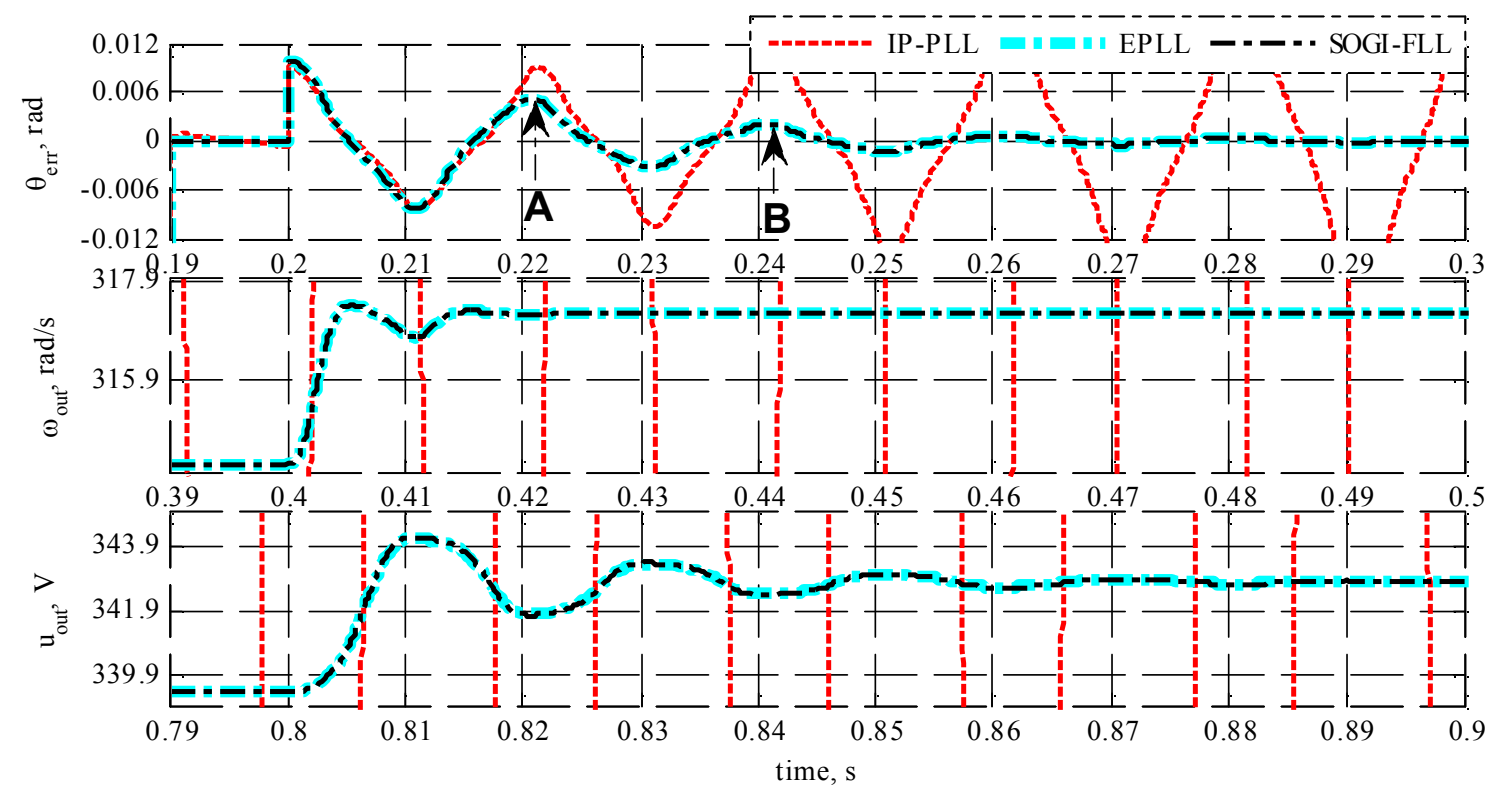

Fig. 8 Simulation results: PLLs testing under small signal step changes for $\mathrm{k}_{\mathrm{v}}=2$. Top subplot: shows phase angle response to a phase-angle step of $0.01 \mathrm{rad}(\mathrm{at} \mathrm{t}=0.2 \mathrm{~s})$, middle subplot: shows frequency response to a frequency step of $1 \%$ (at $\mathrm{t}=0.4 \mathrm{~s}$ ), bottom subplot: shows voltage magnitude response to a voltage step of $1 \%$ (at t=0.8s). "red” IP-PLL, “cyan” EPLL, "black” SOGI-FLL.

From the simulation results for $\theta_{\text {err }}(\delta)$ in (Fig. 8), the slow dynamic PLL eigenvalue that is responsible of the slow decaying oscillation can be approximately determined by measuring the ratio magnitude between two consecutive oscillation peaks $\mathrm{A}\left(\Delta \theta_{\mathrm{A}}=0.005 \mathrm{rad}, \mathrm{t}_{\mathrm{A}}=0.221 \mathrm{~s}\right)$ and $\mathrm{B}\left(\Delta \theta_{\mathrm{B}}=0.002 \mathrm{rad}\right.$, $\left.t_{B}=0.241 \mathrm{~s}\right)$ which results in the position of the eigenvalue on the real axis of $-\ln \left(\Delta \theta_{A} / \Delta \theta_{B}\right) /\left(t_{A}-t_{B}\right) \sim-$ $45.8 \mathrm{~s}^{-1}$. This results in much slower response in comparison to $-314.1 \mathrm{~s}^{-1}$, the desired PLL eigenvalue as emerged from the ZO-DPM based design (14), for $\mathrm{k}_{\mathrm{v}}=2$ (see text above (14)). The problem is that this slow dynamic eigenvalue which is noted by the simulation results of the actual PLL was not possible to be predicted by the ZO-DPM and this is why a higher order DPM is proposed to account for the effect of selected frequency components that might have resulted in such slow dynamic eigenvalue. The DPM developed in general form in $\S 3$ (e.g. for IP-PLL (7)) is customised to $4^{\text {th }}$-order DPM and will be used in the design of the three PLL schemes in the next section. 


\section{Fourth-order Dynamical Phasor Model based Stability Analysis of the PLL Schemes}

\section{Under Investigation}

5.1 Analysis of the $4^{\text {th }}$-order DPM for IP-PLL

The $4^{\text {th }}$-order DPM for the IP-PLL is established using (7), (10). The model is linearized and the small signal eigenvalues are obtained for the equilibrium point $u_{q}=0, u_{d}=U_{v}$. The linearized state space model is of $36^{\text {th }}$ order ( 4 states for the zero-order and 8 states for each "complex" order from 1 to 4 ), which for the sake of maintaining a reasonable paper length, are not detailed. Only four trajectories (TA, TB, TC and TD) for the most dominant complex eigenvalues are plotted in Fig. 9 for $0.52<\mathrm{k}_{\mathrm{v}}<$ 2.1. Four sets of the eigenvalues are highlighted for $\mathrm{k}_{\mathrm{v}}=0.52$ (blue square), 0.92 (cyan star), 1 (red diamond) and 1.12 (green circle) as shown in Fig. 9.

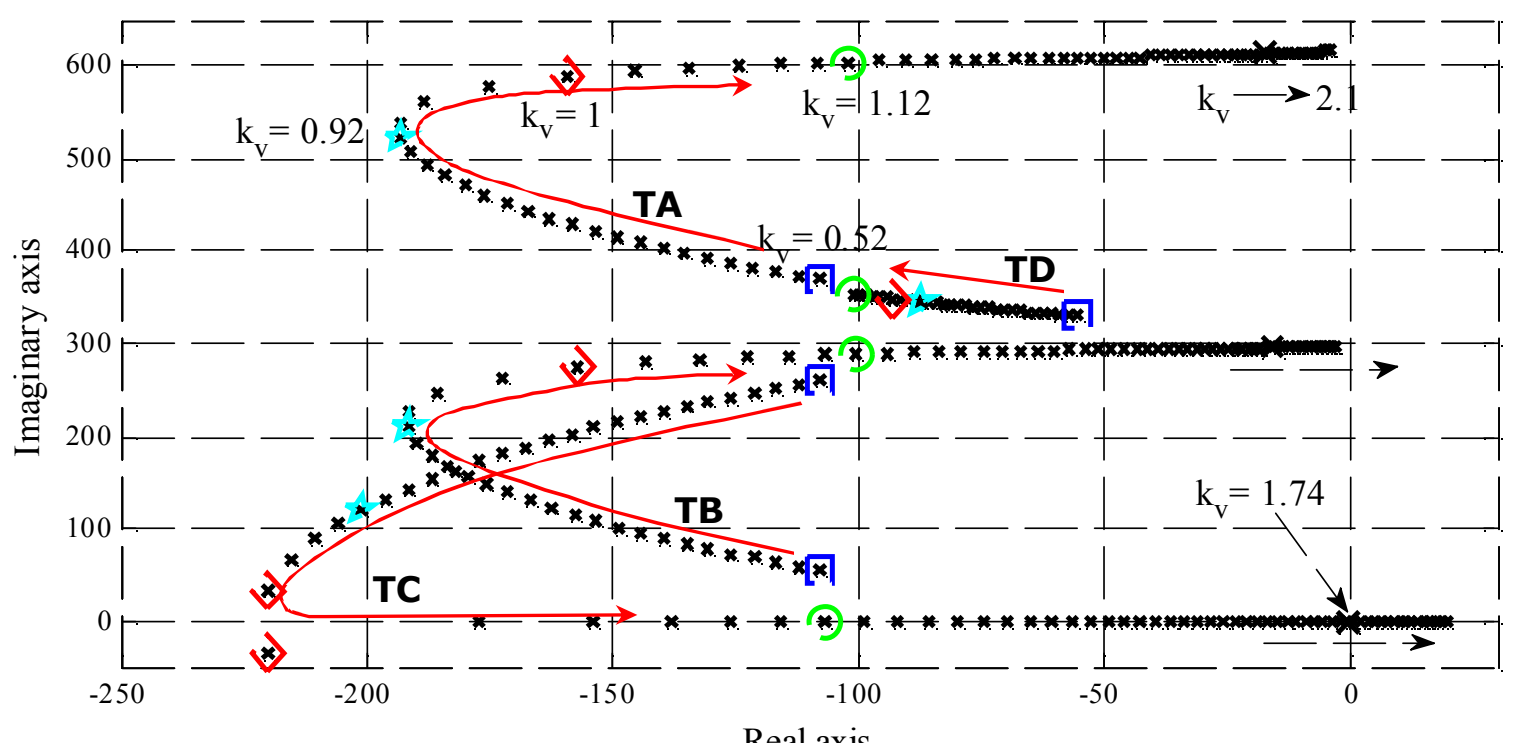

Fig. 9 The four most significant eigenvalue trajectories for the IP-PLL $4^{\text {th }}$ order DPM, $\left(0.52<\mathrm{k}_{\mathrm{v}}<2.1\right)$.

The results show that for $\mathrm{k}_{\mathrm{v}}>1.74$, the eigenvalue TC moves to the right hand plane (instability region), which is consistent with the simulation results presented in Fig. 8 for $\mathrm{k}_{\mathrm{v}}=2$ and in contradiction with the ZO-DPM based small-signal analysis that untruly tells that the PLL is stable for any values of $\mathrm{k}_{\mathrm{v}}$. It is also noted that as $\mathrm{k}_{\mathrm{v}}$ increases from 0.52 to 0.92 , the eigenvalues move further into the left side of the s-plane. The increase of $\mathrm{k}_{\mathrm{v}}$ beyond 0.92 makes three eigenvalue trajectories (TA, TB and TC) to reverse direction towards the unstable side of the s-plane one of which (TC) will tend to cross the stability line first at $\mathrm{k}_{\mathrm{v}}>1.74$. Because the position of one eigenvalue (TD) starts (at 
$\mathrm{k}_{\mathrm{v}}=0.52$ ) significantly on the right side compared to the other three and slowly moves left, whilst the other three start moving right for $\mathrm{k}_{\mathrm{v}}>0.92$, the optimum $\mathrm{k}_{\mathrm{v}}$ is chosen 1.12 so that the position of all four eigenvalues are pushed as left as possible with respect to the real axis to maximize the dynamic response.

\subsection{Analysis of the $4^{\text {th }}$-order DPM for SOGI-FLL}

The $4^{\text {th }}$-order DPM for the SOGI-FLL is derived from (18),(19). The linearized state-space model is of $36^{\text {th }}$ order (not shown to minimise paper length) and the trajectories (TA, TB, TC and TD) of the four most dominant complex eigenvalues are plotted in Fig. 10 for $0.92<\mathrm{k}_{\mathrm{v}}<3.3$. It is found that the eigenvalues for the SOGI-FLL (Fig. 10) are situated more to the left than the eigenvalues for IP-PLL (Fig. 9), which means SOGI-FLL will actually provide better dynamic response than the IP-PLL that contradicts the expected identical dynamic characteristic for all PLLs under study as imposed by the ZO-DPM based design (§3). In Fig. 10, three sets of eigenvalues are highlighted for $\mathrm{k}_{\mathrm{v}}=0.92$ (blue square), 1.3 (red diamond), 1.44 (green circle). This stability analysis based on eigenvalues confirms that the SOGI-FLL is stable for $\mathrm{k}_{\mathrm{v}}<2.82$, which is consistent with the simulation results shown in Fig. 8. Based on the results in Fig. 10, it is recommended that $\mathrm{k}_{\mathrm{v}}<1.44$ to ensure the placement of all the most dominant eigenvalues is situated as far left as possible into the s-plane. The stability limit is found at $\mathrm{k}_{\mathrm{v}}=2.82$.

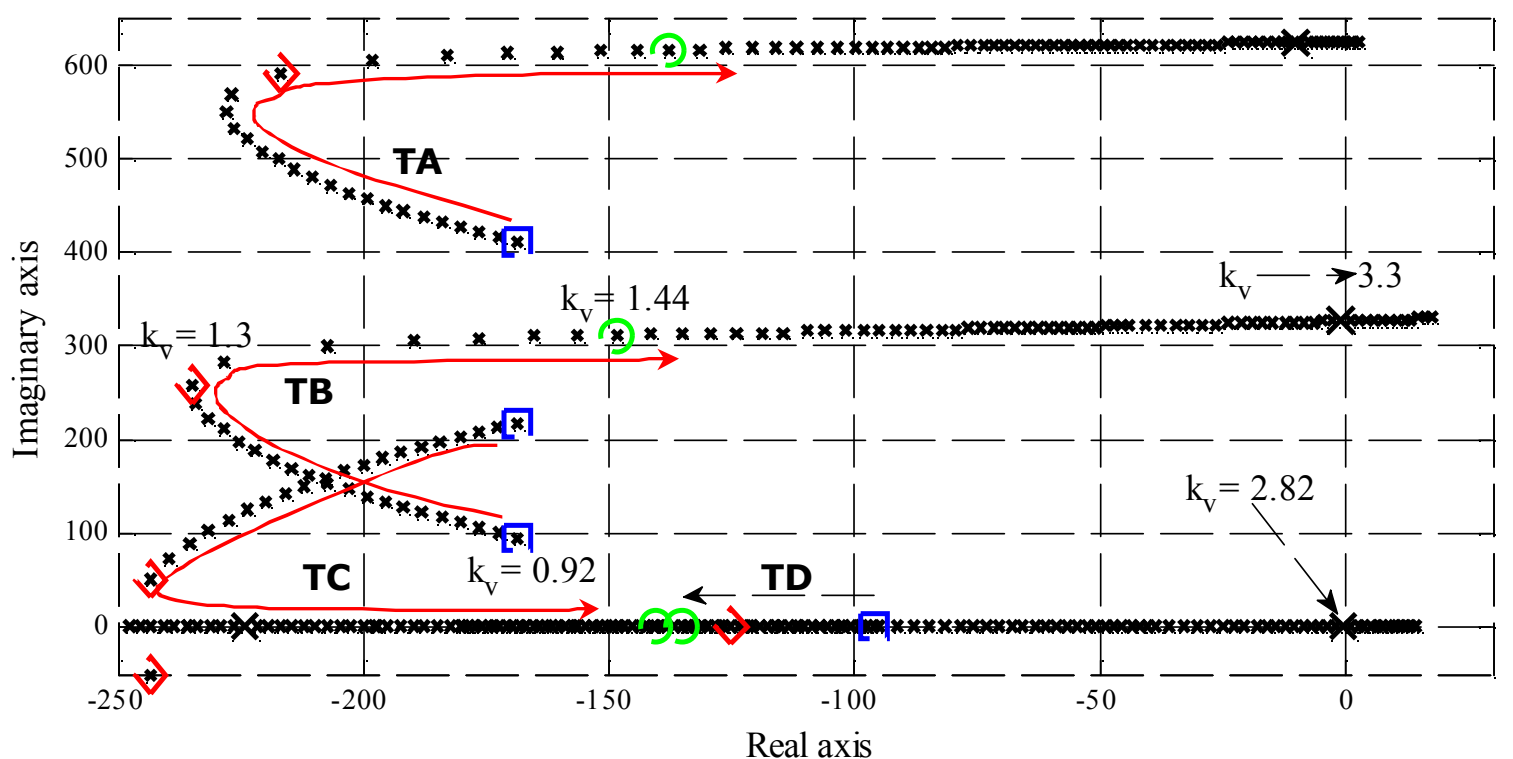

Fig. 10 The four most significant eigenvalue trajectories for the SOGI-FLL $4^{\text {th }}$ order DPM, $\left(0.92<\mathrm{k}_{\mathrm{v}}<3.3\right)$. 


\subsection{Analysis of the $4^{\text {th }}$-order DPM for EPLL}

The state space model for the EPLL (26), (28) is also used to derive the $4^{\text {th }}$-order DPM. The linearized state space model is of $27^{\text {th }}$ order and the trajectories of the four most dominant complex eigenvalues are plotted in Fig. 11 for $0.92<\mathrm{k}_{\mathrm{v}}<3.3$. The EPLL eigenvalue trajectories reveal similar trend to that of the SOGI-FLL. The EPLL is also found unstable for $\mathrm{k}_{\mathrm{v}}>2.82$. Three sets of eigenvalues are highlighted for $\mathrm{k}_{\mathrm{v}}=0.92,1.3,1.44$ as shown in Fig. 11. The trajectories of the EPLL and SOGI-FLL eigenvalues are almost the same as revealed by comparing Figs 10 and 11. Therefore, the recommended value for $\mathrm{k}_{\mathrm{v}}$ is equal to that for SOGI-FLL, i.e. $\mathrm{k}_{\mathrm{v}}<1.44$.

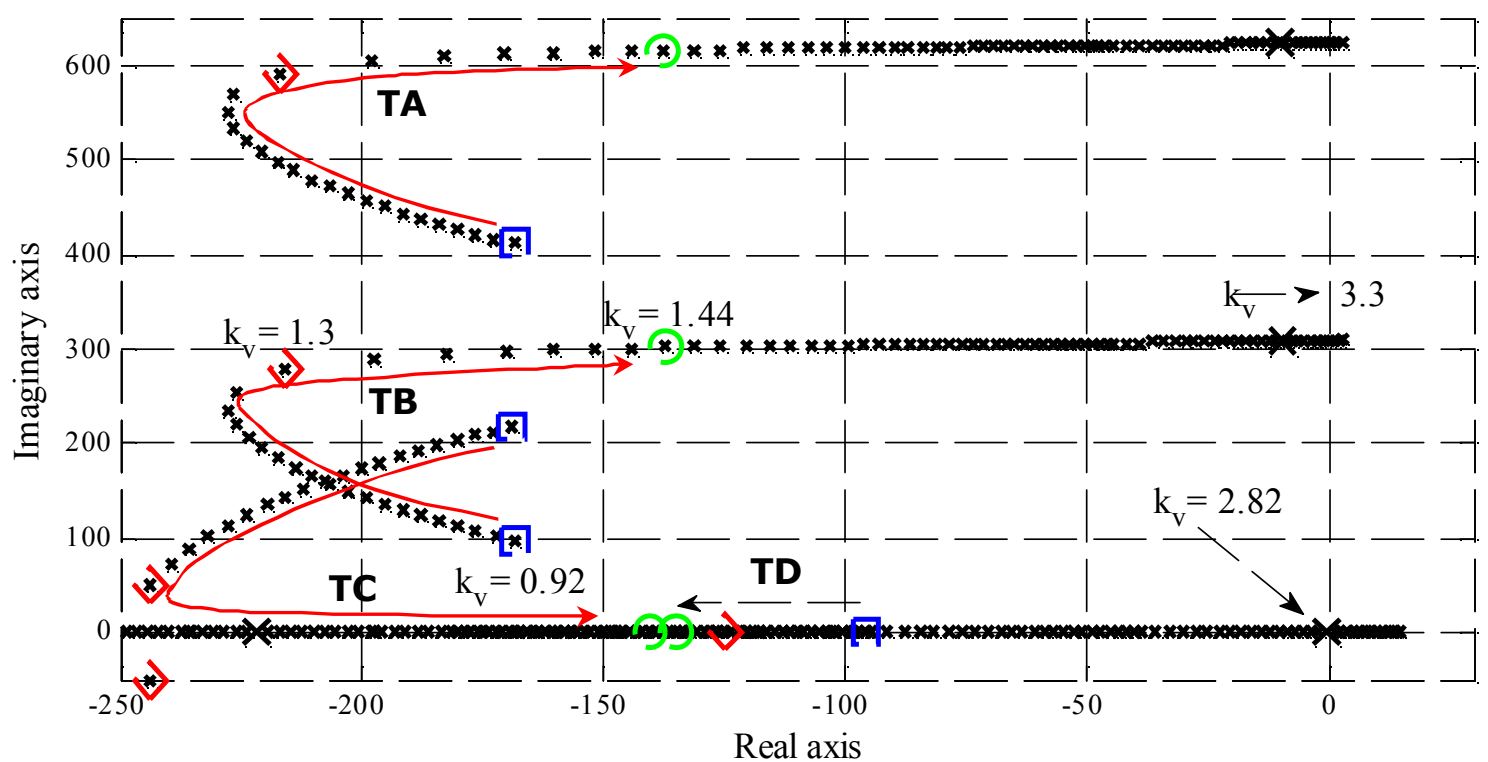

Fig. 11 The four most significant eigenvalue trajectories for the EPLL $4^{\text {th }}$ order DPM, $\left(0.92<\mathrm{k}_{\mathrm{v}}<3.3\right)$.

\subsection{Validation by Simulation of the $4^{\text {th }}$-order DPMs}

The analysis of the eigenvalues presented in the previous sections can be summarised in Table 1 which contains the values of $\mathrm{k}_{\mathrm{v}}$ for operation at stability limit (top), for keeping all of the most dominant eigenvalues as far left on the s-plane as possible as a design limit (middle) and a set of gains selected in the paper (bottom) that agree with both previous limitations and were recommended to be used in the large signal tests.

Table 1: Gain $\mathrm{k}_{\mathrm{v}}$ design values for the three PLLs under study.

\begin{tabular}{|c|c|c|c|}
\hline & IP-PLL & SOGI-FLL & EPLL \\
\hline Stability limit & $\mathrm{k}_{\mathrm{v}}<1.72$ & $\mathrm{k}_{\mathrm{v}}<2.82$ & $\mathrm{k}_{\mathrm{v}}<2.82$ \\
\hline Design limit & $\mathrm{k}_{\mathrm{v}}<1.12$ & $\mathrm{k}_{\mathrm{v}}<1.44$ & $\mathrm{k}_{\mathrm{v}}<1.44$ \\
\hline Recommended in this paper & $\mathrm{k}_{\mathrm{v}}=1$ & $\mathrm{k}_{\mathrm{v}}=1.3$ & $\mathrm{k}_{\mathrm{v}}=1.3$ \\
\hline
\end{tabular}


The eigenvalues analysis of the $4^{\text {th }}$-order DPM of the PLL schemes under study is validated by simulation. Fig. 12 illustrates the simulation results of the three PLL schemes for $k_{v}=2.82$ subjected to small step changes in phase angle, frequency and voltage magnitude. All three tests show that the IP-PLL is unstable while the SOGI-FLL and the EPLL response were both on the verge of instability. These findings validate the obtained stability limits from the small signal eigenvalues analysis shown in Figs 9-11 and hence prove the suitability of the $4^{\text {th }}$-order DPM for PLL stability analysis and control design.

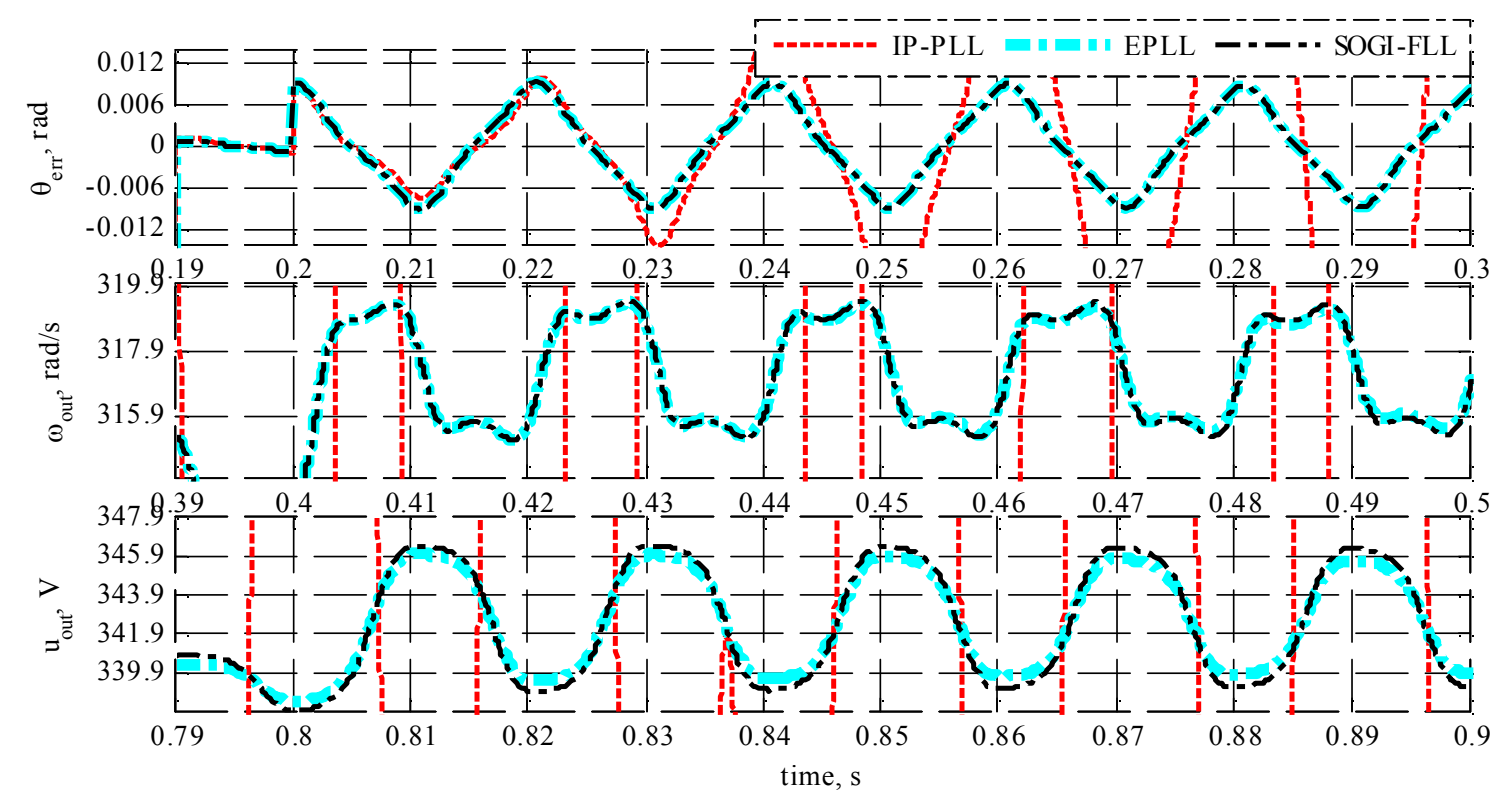

Fig. 12 Simulation results: PLLs testing under small signal step changes for $\mathrm{k}_{\mathrm{v}}=2.82$. Top subplot: shows phase angle response to a phase-angle step of $0.01 \mathrm{rad}($ at $\mathrm{t}=0.2 \mathrm{~s})$, middle subplot: shows frequency response to a frequency step of $1 \%$ (at $\mathrm{t}=0.4 \mathrm{~s}$ ), Bottom subplot: shows voltage magnitude response to a voltage step of $1 \%$ (at t=0.8s). "red” IP-PLL, “cyan” EPLL, "black” SOGI-FLL.

In the next section, the three PLL schemes using the recommended design values for $\mathrm{k}_{\mathrm{v}}$ in Table 1 will be tested and compared for large signal disturbances.

\section{Large Signal Testing and Performance Comparison of the PLL Schemes}

The models for the PLL schemes in Fig. 2, 4, 6 using the design adaptive laws derived in (10), (19) and (27) will be tested by simulation and experimental implementation for large signal disturbances. The recommended values for $\mathrm{k}_{\mathrm{v}}$ listed in the last row of Table 1 are used in both simulations and experiments. The disturbances applied are: phase jump $\left(\Delta \theta_{v}=1 \mathrm{rad}\right)$, voltage sag $\left(\Delta U_{v}=80 \%\right.$ of the nominal value) and frequency step change $\left(\Delta \omega_{v}=10 \%\right.$ of the nominal value). Large disturbance 
in grid voltage can cause the PLLs to slip and loose the locking state for one or more cycles. The locking state is maintained attractive under large disturbance by limiting $\omega_{\mathrm{e}}$ to be within a band of $\pm 30 \%$ from the nominal-value such that $0.7 \omega_{\mathrm{n}}<\omega_{\mathrm{e}}<1.3 \omega_{\mathrm{n}}$. Also, the absolute value of $\mathrm{u}_{\mathrm{d}}$ is used in the denominator of the adaptive laws (10) and (27). The simulation models are implemented in Matlab/Simulink. The models of the three PLL schemes are also implemented on a 32-bit floating point DSP+FPGA laboratory digital platform equipped with 16 bit $\mathrm{A} / \mathrm{D}$ converters specially designed for real time control of power electronic systems. All three PLL schemes were running simultaneously and independently in the DSP with a sampling time of $100 \mu \mathrm{s}$. A programmable electronic AC power source (Chroma) is used to generate the various types of grid voltage disturbances needed for the experimental validation such as phase jump, voltage sag and frequency step change tests. Because of the existence of an LC filter on the output of the electronic power supply, there is a limitation of how fast/sharp the voltage transients can be replicated and this can explain some of the differences that will be seen in the next subsections between the simulation and the experimental results.

The DSP control algorithm which is executed every sampling time starts by acquiring the supply voltage, then independently calculates the state variables for all three PLL schemes; at the end of every sampling time, all state variables (including the measured supply voltage) are saved into a memory buffer with a sufficient length to store the full response to the disturbance. The content of this memory buffer is later transferred to the PC for visualisation. There is no post processing of data. The simulation and experimental results of the three PLL schemes under investigation will be compared in the following sections. The simulation results are shown in the left subplots of following figures while the corresponding experimental results are shown in the right subplots.

\subsection{Response Following the Phase Jump Test}

The PLLs are tested for large and sudden phase jump of $1 \mathrm{rad}$. The PLLs with the $\pm 30 \% \omega_{\mathrm{e}}$ limit are tested for phase jump response. The results in Fig. 13 show that all three PLL schemes are stable for a large phase jump disturbance. It is noted that the SOGI-FLL phase tracking is faster simply because the phase angle is estimated using the "arctangent" function of the estimated voltage vector (22) rather 
than by direct integration of $\omega_{\mathrm{e}}$ which is subject to the $\pm 30 \%$ limit. On the other hand, the EPLL and IP-PLL have experienced slow phase-angle tracking responses because of the limits imposed to $\omega_{\mathrm{e}}$ that is fed to the integrator. It is also noted that SOGI-FLL has provided smaller disturbance to the estimated voltage magnitude (see bottom subplots of Fig. 13) during the phase jump. The results show that SOGI-FLL could be the most suitable choice for grids that suffer from frequent phase jumps.
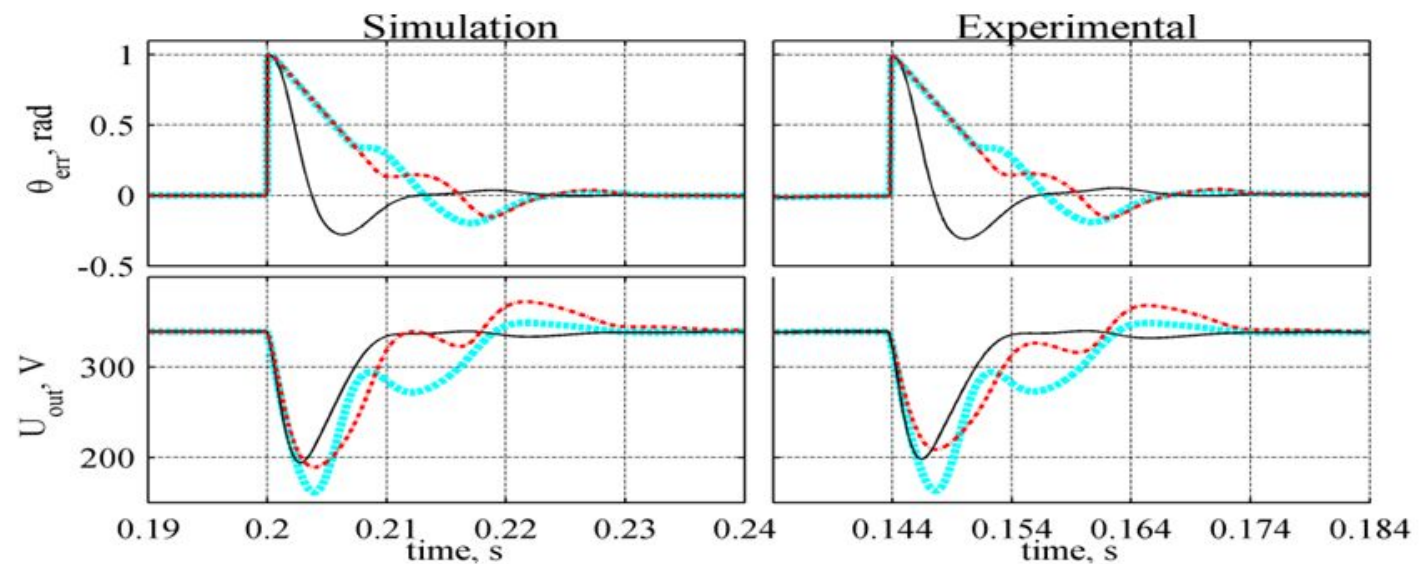

Fig. 13 Response of the three PLL schemes to phase jump: (Top subplots) phase angle response, (bottom subplots) voltage magnitude response. "red" is for IP-PLL, "cyan" is for EPLL and "black" is for SOGI-FLL.

\subsection{Response Following the Voltage Sag Test}

Modern grid codes require the converters to continue operation even under severe voltage sags to support the grid recovery by injecting reactive current. This will require PLLs to maintain tracking of the grid voltage vector trajectory with minimal error in phase angle estimation. Typically, the voltage sag transient that signals the beginning of a grid fault is the sharpest whilst the grid voltage recovery is a much slower process (slow ramp). The designed PLLs are tested for a large voltage sag of $80 \%$ applied at the zero crossing of grid voltage (worst case for voltage estimation) and setting the limit for the estimated angular frequency as $0.7 \omega_{\mathrm{n}}<\omega_{\mathrm{e}}<1.3 \omega_{\mathrm{n}}$. The simulation and the experimental results are shown in Fig 14. The voltage tracking of all three PLL schemes is good with no cycle slip. The EPLL have shown the faster voltage magnitude response to the step voltage change. The phase angle error for SOGI-PLL was the largest. 

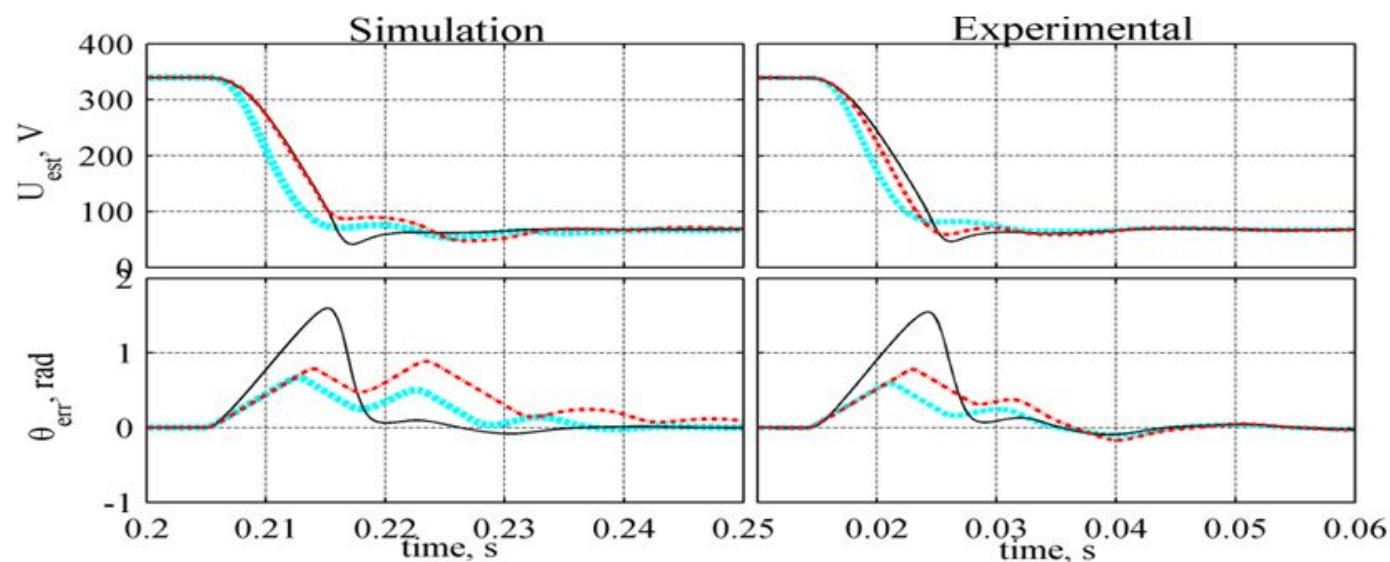

Fig. 14 Response of the three PLL schemes to $80 \%$ voltage sag test; (top subplots) voltage magnitude response, (bottom subplots) phase angle response. "red" is for IP-PLL, "cyan" is for EPLL and "black" is for SOGI-FLL.

\subsection{Response Following the Frequency Step Change Test}
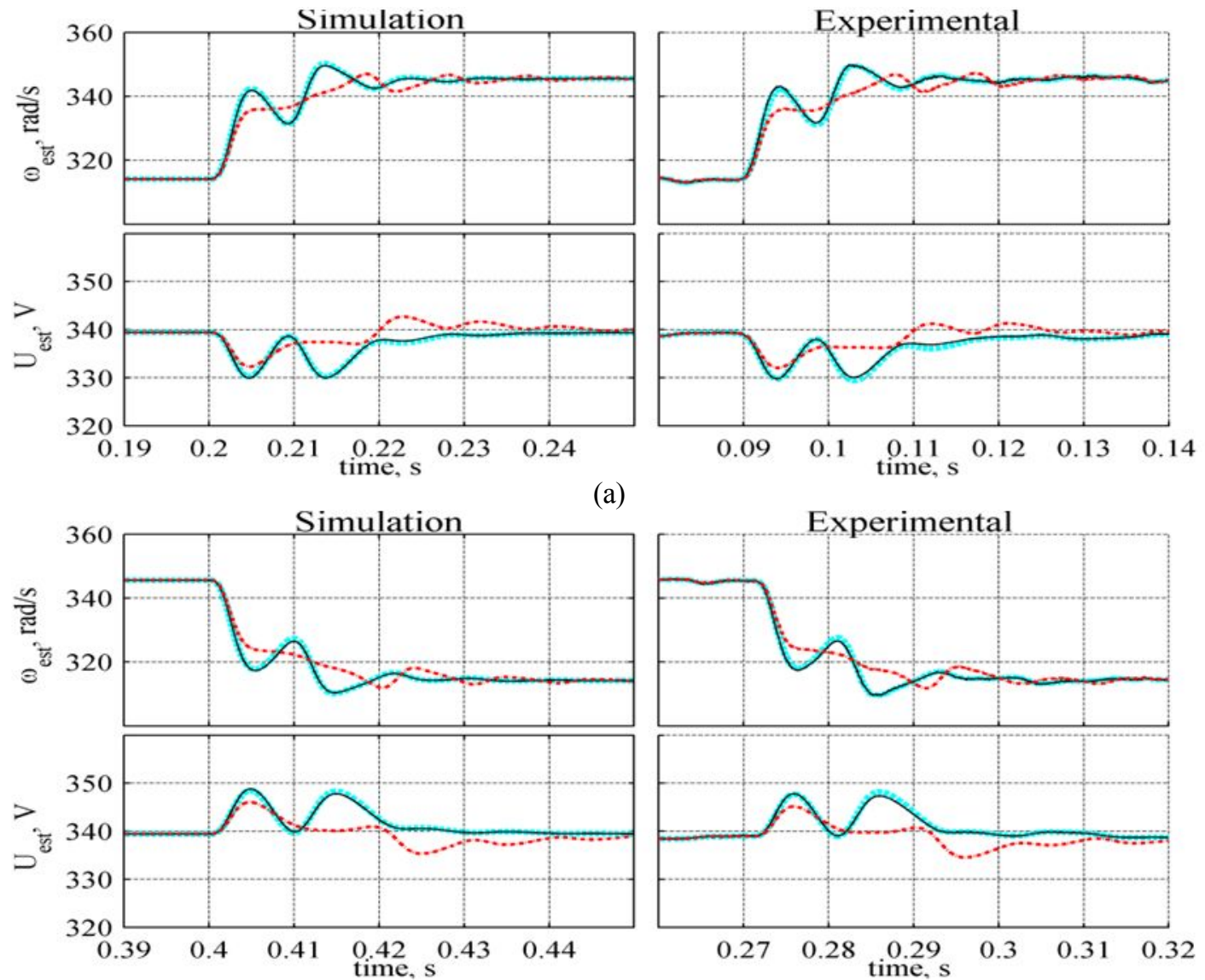

(b)

Fig. 15 Response of the three PLL schemes to frequency step change test: a) frequency step increase results, b) frequency step decrease results. (top subplots) frequency response, (bottom subplots) voltage magnitude response. "red" is for IP-PLL, "cyan" is for EPLL and "black" is for SOGI-FLL.

The PLL schemes are tested for a sudden change in grid frequency. The frequency is changed by applying a $\pm 10 \%$ step of the nominal value $(50 \mathrm{~Hz})$. The simulation and the experimental results are shown in Fig. 15a,b. The results show that all PLL schemes are stable but whilst the EPLL and SOGI- 
FLL have nearly identical response, the IP-PLL has a slightly slower response. The error in the voltage estimation of the IP-PLL was the smallest.

The conclusion of these tests is that all three PLL schemes perform well under all three large signal disturbances. The SOGI-FLL was able to maintain its good phase angle dynamic response during the phase jump test because the output phase angle is calculated directly from the PLL output voltage vector using arctangent. However, during the voltage sag test which would result in errors in the estimated voltage, it results in the largest phase angle error. The responses of the three PLL schemes to a step change in grid frequency were similar, but with slightly slower dynamics for IP-PLL.

\section{Conclusion}

The use of Dynamic Phasor Modelling DPM is proposed in this paper to improve the modelling for the purpose of stability analysis and design of three PLL schemes, the single-phase IP-PLL, SOGIFLL and EPLL PLL. First, the simplified average model usually used in the literature for single phase PLL design and stability analysis has been used to design three PLL schemes to achieve identical dynamic characteristics which when evaluated via simulation, are found to differ significantly.

For this reason, fourth-order DPMs have been developed for the three PLL schemes under study. An analysis of the most predominant eigenvalues is used to determine the stability limits and the recommended design gains which are then validated via simulation for small signal disturbances. The actual small-signal dynamic response of the PLLs was as predicted by the $4^{\text {th }}$-order DPM eigenvalue analysis.

The final validation of the $4^{\text {th }}$-order DPM based design of the PLL schemes is achieved by large signal disturbance testing (phase jump, voltage sag and frequency step change) implemented both in simulation and on an experimental digital control platform using (as input) actual voltage disturbances produced by a programmable electronic AC power supply. The SOGI-FLL is found to be more suitable for operation under severe phase jump situations. EPLL on the other hand, had the best response during voltage sag. 


\section{Appendix 1}

\section{Dynamic Phasor Modelling of Non-linear State Variables}

The DPM of the nonlinear terms such as $\cos (2 \theta)$ and $\sin (2 \theta)$, e.g. in $(5 \mathrm{a}, \mathrm{b})$ are given by:

$$
\begin{aligned}
& \langle\sin (m \theta)\rangle_{k}=\left\{\begin{array}{c}
\mp \frac{1}{2} j \quad \ldots \ldots \text { for } k= \pm m \\
0 \ldots \ldots \ldots \text { otherwise }
\end{array}\right. \\
& \langle\cos (m \theta)\rangle_{k}=\left\{\begin{array}{l}
\frac{1}{2} \ldots \ldots \text { for } k=\mp m \\
0 \ldots \ldots \ldots \text { otherwise }
\end{array}\right.
\end{aligned}
$$

For the nonlinear cosine and sine terms function of $\theta$ and $\delta$ in $(5 a, b)$, Taylor expansion method is applied before using the convolution property (4) to determine the DPM. The Taylor expansion for the various nonlinear terms in $(5 \mathrm{a}, \mathrm{b})$ assuming $\delta$ is small are expressed as:

$$
\begin{gathered}
\cos (\delta) \sim 1 \quad ; \quad \sin (\delta) \sim \delta \\
\cos (\delta+2 \theta) \sim \cos (2 \theta)-\delta \sin (2 \theta) \\
\sin (\delta+2 \theta) \sim \sin (2 \theta)+\delta \cos (2 \theta)
\end{gathered}
$$

Then, the Fourier coefficients for the nonlinear terms in (A2) are derived by applying rules (4) and (A1):

$$
\begin{aligned}
& \langle\cos (\delta)\rangle_{0}=1 ;\langle\cos (\delta)\rangle_{k \neq 0}=0 \\
& \langle\sin (\delta)\rangle_{k}=\langle\delta\rangle_{k} \\
& \langle\cos (\delta+2 \theta)\rangle_{2}=0.5+0.5 j\langle\delta\rangle_{0}-0.5 j\langle\delta\rangle_{-4} \quad \text { and } \quad\langle\cos (\delta+2 \theta)\rangle_{k \neq 2}=0 \\
& \langle\sin (\delta+2 \theta)\rangle_{2}=-0.5 j+0.5 j\langle\delta\rangle_{0}+0.5\langle\delta\rangle_{-4} \quad \text { and } \quad\langle\sin (\delta+2 \theta)\rangle_{k \neq 2}=0
\end{aligned}
$$

The nonlinear term $\left(u_{q} / u_{d}\right)$ in (e.g. in (10), (27)) is approximated by assuming $u_{d}$ is mainly a dc quantity with additional small ripple component, (Emadi, 2004) (i.e. $u_{d}=\left\langle u_{d}\right\rangle_{0}+\tilde{u}_{d}$ ) and hence (using Taylor expansion method):

$$
\frac{u_{q}}{u_{d}} \sim u_{q}\left[\frac{1}{\left\langle u_{d}\right\rangle_{0}}-\frac{\tilde{u}_{d}}{\left(\left\langle u_{d}\right\rangle_{0}\right)^{2}}\right] \sim u_{q}\left[\frac{2}{\left\langle u_{d}\right\rangle_{0}}-\frac{u_{d}}{\left(\left\langle u_{d}\right\rangle_{0}\right)^{2}}\right]
$$

where $\tilde{u}_{d}$ is the sum of the ripple components and $\left\langle\mathrm{u}_{d}\right\rangle_{0}$ is the DC component of $u_{d}$. Then, the convolution property (4) is applied to (A4) to give:

$$
\left\langle\frac{u_{q}}{u_{d}}\right\rangle_{k} \sim\left[\frac{2}{\left\langle u_{d}\right\rangle_{0}}\left\langle u_{q}\right\rangle_{k}-\frac{1}{\left(\left\langle u_{d}\right\rangle_{0}\right)^{2}}\left\langle u_{q} u_{d}\right\rangle_{k}\right]
$$




\section{References}

Caliskan, V.A., Verghese, G.C. and Stankovi'c, A.M. (1999), "Multifrequency Averaging of DC/DC Converters", IEEE Transactions on Power Electronics, Vol. 14, No. 1, pp. 124-133.

Chung Se-Kyo (2000), “A Phase Tracking System for Three Phase Utility Interface Inverters”, IEEE Transactions on Power Electronics, Vol. 15, No. 3, pp. 431-438.

De Brabandere, K., Bolsens, B., Van den Keybus, J., Driesen, J., Prodanovic, M. and Belmans, R. (2005), "Small-signal stability of grids with distributed low-inertia generators taking into account line phasor dynamics", 18th International Conference and Exhibition on Electricity Distribution, CIRED, Vol., No., 6-9 June, pp. 1-5.

Emadi, A. (2004), "Modeling of Power Electronic Loads in AC Distribution Systems Using the Generalized State-Space Averaging Method", IEEE Transactions on Industrial Electronics, Vol. 51, No. 5, pp. 992-1000.

Filho, R.M.S., Seixas, P.F., Cortizo, P.C., Torres, L.A.B. and Souza, A.F. (2008), "Comparison of three single-phase PLL algorithms for UPS applications", IEEE Transactions on Industrial Electronics, Vol. 55, No. 8, pp. 2923-2932.

Freijedo, F.D., Doval-Gandoy, J., López, Ó. and Acha, E. (2009), “Tuning of Phase-Locked Loops for Power Converters Under Distorted Utility Conditions", IEEE Transactions on Industry Applications, Vol. 45, No. 6, pp. 2039-2047.

Golestan, S., Monfared, M., Freijedo, F. D. and Guerrero, J. M. (2013), "Advantages and Challenges of a Type-3 PLL", IEEE Transactions on Power Electronics, Vol. 28, No. 11, pp. 4985-4997.

Karimi-Ghartemani, M. (2013), "A Unifying Approach to Single-Phase Synchronous Reference Frame PLLs", IEEE Transactions on Power Electronics, Vol. 28, No. 10, pp. 4550-4556.

Karimi-Ghartemani, M., Khajehoddin, S.A., Jain, P.K. and Bakhshai, A. (2012), "Problems of startup and phase jumps in PLL systems", IEEE Transactions on Power Electronics, Vol. 27, No. 4, pp. 18301838.

Mariani, V., Vasca, F. and Guerrero, J.M. (2014), "Dynamic-phasor-based Nonlinear Modelling of AC Islanded Microgrids under Droop Control", 11th International Multi-Conference on Systems, Signals \& Devices (SSD), Vol., No., 11-14 Feb., pp.1-6.

Mattavelli, P., Stankovi, A.M. and Verghese, G.C. (1999), "SSR Analysis with Dynamic Phasor Model of Thyristor-Controlled Series Capacitor" IEEE Transactions on Power Systems, Vol. 14, No. 1, February 1999, pp. 200-208.

Nicastri, A. and Nagliero, A. (2010) "Comparison and evaluation of the pll techniques for the design of the grid-connected inverter systems", in Proc. IEEE Int. Symp. Ind. Electron., pp. 3865-3870.

Rashed, M., Klumpner, C. and Asher, G. (2013), "Repetitive and Resonant Control for a Single-Phase Grid-Connected Hybrid Cascaded Multilevel Converter", IEEE Transactions on Power Electronics, Vol. 28, No. 5, pp. 2224- 2234.

Rodr'iguez, P., Luna, A., Candela, I., Mujal, R.,Teodorescu, R. and Blaabjerg, F. (2011), "Multiresonant frequency-locked loop for grid synchronization of power converters under distorted grid conditions", IEEE Transactions on Industrial Electronics, Vol. 58, No. 1, pp. 127-138.

Sanders, S.R., Noworolski, J.M., Liu, X.Z. and Verghese, G.C. (1991), "Generalized Averaging Method for Power Conversion Circuits", IEEE Transactions on Power Electronics, Vol. 6. No. 2., pp. 251-259

Shinnaka, S. (2011), "A novel fast-tracking d-estimation method for single-phase signals", IEEE Transactions on Power Electronics, Vol. 26, No. 4, pp. 1081-1088.

Silva, S., Lopes, B., Campana, R. and Bosventura, W. (2004), "Performance evaluation of PLL algorithms for single-phase grid-connected systems", $39^{\text {th }}$ IEEE IAS Annual Industry Applications Conference Proceeding, Oct., Vol. 4, pp. 2259-2263. 
Stankovic, A.M., Lesieutre, B.C. and Aydin, T. (1999), "Modeling and Analysis of Single-Phase Induction Machines with Dynamic Phasors", IEEE Transactions on Power Systems, Vol. 14, No. 1, pp. 9-14.

Thacker, T., Boroyevich, D., Burgos, R. and Wang, F. (2011), "Phase-Locked Loop Noise Reduction via Phase Detector Implementation for Single-Phase Systems", IEEE Transactions on Industrial Electronics, Vol. 58, No. 6, pp. 2482-2490.

Velasco, D., Trujillo, C., Garcera, G. and Figueres, E. (2011), "An active anti-islanding method based on phase-PLL perturbation", IEEE Transactions on Power Electronics, Vol. 26, No. 4, pp. 1056-1066.

Wang, L., Guo, X. Q., Gu, H. R., Wu, W.Y. and Guerrero, J.M. (2012), "Precise Modeling based on Dynamic Phasors for Droop-controlled Parallel-connected Inverters", IEEE International Symposium on industrial Electronics (ISIE), Vol., No., 28-31 May, pp. 475-480.

Xianwei, W., Fang Z., Haiping, G., Liang, M, Meijuan, Y. and Jinjun L. (2011), "Stability analysis of droop control for inverter using dynamic phasors method", IEEE Energy Conversion Congress and Exposition (ECCE), Vol., No., 17-22 Sept., pp. 739-742.

\begin{abstract}
About the authors
Dr Mohamed Rashed received the PhD Degree in Electric Motor Drives from the University of Aberdeen, Aberdeen, UK, in 2002. From 2002 to 2005, he was a Postdoctoral Fellow at the Department of Engineering, the University of Aberdeen. He is an Associate Professor at the Department of Electrical Engineering, the Mansoura University, Egypt and currently is on leave working as a Research Fellow, Power electronics, Machines and Control Group, Department of Electrical and Electronic Engineering, The University of Nottingham, Nottingham, UK His research interests include the design and control of electric motor drive systems and power electronics for micro grids, renewable energy sources, and energy storage systems. Dr Mohamed Rashed is the corresponding author and can be contacted at: mohamed.rashed@nottingham.ac.uk
\end{abstract}

Dr Christian Klumpner received the PhD Degree in Electrical Engineering from the "Politehnica" University of Timisoara, Timisoara, Romania, in 2001. From 2001 to 2003, he was a Research Assistant Professor in the Institute of Energy Technology, the Aalborg University, Aalborg, Denmark. From October 2003 to 2011, he was a Lecturer with the Department of Electrical Engineering, University of Nottingham, Nottingham, UK, where he is currently an Associate Professor. His current research interests include power electronics for various applications such as AC drives, connecting renewable energy sources and energy storage devices to the AC power grid. Dr Klumpner received the Isao Takahashi Power Electronics Award in 2005 at the International Power Electronics Conference organised by the Institute of Electrical Engineers of Japan in Niigata. He is also a recipient of the 2007 IEEE Richard M. Bass Outstanding Young Power Electronics Engineer Award.

Professor Greg Asher received the BSc and PhD Degrees from the Bath University, Bath, England, in 1976. He was a Research Fellow in superconducting systems at the University of Bangor, Wales, UK. He was a Lecturer in Control at the University of Nottingham, Nottingham, UK, in 1984, where he developed an interest in motor drive systems, particularly the control of AC machines. He was a Professor of Electrical Drives in 2000, the Head of the School of Electrical and Electronic Engineering in 2004, and an Associate Dean for Teaching and Learning in the Engineering Faculty in 2008. He has published nearly 300 research papers, has received over $£ 5 \mathrm{M}$ in research contracts, and has successfully supervised $31 \mathrm{PhD}$ students. His research interests include motor drive control, powersystem modelling, power microgrid control, and aircraft power systems. Professor Asher was a Member of the Executive Committee of European Power Electronics Association until 2003, Chair of the Power Electronics Technical Committee for the Industrial Electronics Society until 2008, and is an Associate Editor of the IEEE Industrial Electronics Society. 